\title{
Pharyngeal Anastomotic Leakage
}

National Cancer Institute

\section{Source}

National Cancer Institute. Pharyngeal Anastomotic Leakage. NCI Thesaurus. Code C78550.

Leakage due to breakdown of a pharyngeal anastomosis. 